\title{
Comparison of the effect of ginger capsule and diclofenac tablet on the relief of pain following inguinal hernia surgery
}

\author{
Gholamreza Shabanian $^{1 *}$, Ali Satari ${ }^{2}$ \\ 'Department of Anesthesia, Shahrekord University of Medical Sciences, Shahrekord, Iran \\ ${ }^{2}$ Student Research Committee, Shahrekord University of Medical Sciences, Shahrekord, Iran
}

*Corresponding Author: Gholamreza Shabanian, Department of Anesthesia, Ayatollah Kashani Hospital, Shahrekord University of Medical Sciences, Iran, Tel: +989133827110, E-mail: gshabanian@yahoo.com

\begin{abstract}
Background and aims: Postoperative pain control decreases negative effects on the health domains of the patients and their relatives in addition to reducing the costs and the duration of hospitalization. The aim of the present study was to comparatively investigate the analgesic effects of ginger and diclofenac tablets.

Materials and Methods: In this clinical trial, 80 patients undergoing inguinal hernia surgery were randomly divided into two groups of 40. One group received $250 \mathrm{mg}$ ginger capsule while the other one received $100 \mathrm{mg}$ diclofenac tablet. Then, the patients were examined within 6 hours (every two hours) and their pain was assessed using the standard 10-point visual analogue scale. Finally, the data were analyzed using the SPSS, version 22.

Results: Based on the results, there was no significant difference in pain severity between the two groups at $0,2,4$, and 6 hours after recovery $(P>0.05)$. In addition, no significant difference was observed in the duration of pain since the time of patient recovery between the two groups $(P>0.05)$. Further, as regards the number of patients receiving pethidine, no difference was found between the two groups $(P>0.05)$.

Conclusion: In general, both ginger capsule and diclofenac tablet were similarly effective in relieving the pain after inguinal hernia surgery. Keywords: Inguinal hernia, Ginger, Diclofenac, Pain
\end{abstract}

Received: 6 December 2018, Accepted: 23 February 2019, ePublished: 24 June 2019

\section{Introduction}

Pain is one of the major public health issues around the world that imposes heavy economic burden on the community (1-4). In addition to bothering the patient, pain can impair the quality of life and thus adversely affect the family and social aspects of the patients' lives (5). Therefore, pain relief in patients reduces the adverse effects of pain on the patient's physical and mental conditions and provides comfort for his/her family (6). Postoperative pain relief, especially through certain regimens, can decrease mortality during and after the operation $(7,8)$. Pain control with the least possible complications, as one of the dimensions of patient satisfaction with health care services, is one of the goals of pain management (6). There are several ways to reduce pain in patients. For example, nonsteroidal anti-inflammatory drugs and opioids can be used to treat various pains, including surgical pain (9).

Meanwhile, herbal remedies have drawn attention because of their cost-effectiveness and fewer side effects in long-term use, including chronic pains (10-14). Plants can even be used as food supplements and be effective in reducing the pain by inducing anti-inflammatory properties (15-18). Zingiber officinale rhizome is one of the oldest medicinal and preventive remedies for many conditions, including pain (19-23). However, despite the anti-inflammatory effects of ginger, its effect on pain relief is controversial (24). Therefore, the present study was conducted to compare the effects of the ginger capsule and diclofenac tablet on the relief of pain following inguinal hernia surgery.

\section{Materials and Methods}

In this prospective double-blind clinical trial, a total of 80 patients submitted to inguinal hernia repair surgery in Kashani hospital of Shahrekord were selected by convenience sampling technique. The sample size was calculated at 40 for each group using a sample size statistics formula and according to the protocol of a similar study (25). The inclusion criteria were those patients aged over 14 years who were eligible for inguinal hernia repair surgery and provided consent to participate in the study. In addition, the exclusion criteria included over 1-hour duration of surgery, classified as higher than ASA (American Association of Analgesia) II, a history of cardiovascular, liver, kidney, pulmonary, as well as psychiatric diseases, epilepsy, and diseases affecting the function of the nervous 
and muscular system, addicted or alcoholic or the habitual use of any related drug, emergency surgery, gastrointestinal problems, the unintentional division of ilioinguinal nerve and/or iliohypogastric nerve during the surgery, receiving analgesics 24 hours before surgery, asthma, eczema or hypersensitivity to ginger, recurrent hernia, neurological lesions such as stroke, nerve division due to trauma and congenital neurological diseases, and the lack of ability to communicate verbally and express the pain. The data collection instrument was a checklist, the first section of which consisted of demographic characteristics such as age, a history of underlying diseases, hospitalization and surgery records, associated symptoms including nausea and vomiting, as well as postoperative complications (up to 10 days) including infection and hematoma after taking the studied drugs. The second section addressed pain severity and the duration of postoperative pain that was measured by a standard 10-point visual analogue scale (26). Anesthesia was recorded on a 10-point (1-10) scale, with the same visual analogue scale form used at all intervals, and therefore the patient would select the choice that indicated the severity of his/her pain. Before entering the operating room, patients received no pretreatment and were divided into two groups.

In order to control postoperative pain, the patients in the ginger group received one ginger capsule (Zintoma) orally with $50 \mathrm{~mL}$ water one hour before surgery, while the diclofenac group received diclofenac $100 \mathrm{mg}$ with 50 $\mathrm{mL}$ water. Further, both groups received $2 \mathrm{mg}$ midazolam one hour before the surgery. In recovery, pulse oximetry was monitored and oxygen was given via the facial mask. The first examination of the pain was conducted within 6 hours (once every two hours) after complete recovery in the recovery room and after the patient was transferred to the department. Furthermore, the pain severity was assessed by the visual analogue scale criteria while the incidence of vomiting, respiratory depression, urinary retention, shivering, and hemodynamic changes, as well as the amount and type of injectable drug were monitored by a contributor to the research project who was blind to the type of the drug prescribed for the patient. The duration of the pain for all patients was considered to begin from the time the patient recovered to when the pain severity decreased to less than three.

In addition, $25 \mathrm{mg}$ of pethidine was injected to the patient if requested or needed (pain score higher than 5) and the number of injections of pethidine was recorded.

The obtained data were analyzed by SPSS, version 22. The frequency and relative frequency were used for qualitative variables and mean (standard deviation) was employed for quantitative variables. Moreover, qualitative variables were analyzed by chi-squared and Fisher exact tests and the mean values in the two groups were compared by using Student's $t$ test or its nonparametric equivalent (Mann-Whitney test) depending on data distribution.
$P<0.05$ was considered statistically significant.

Results

The mean age of the participants in the ginger and diclofenac groups was $41.47 \pm 14.44$ and $44.22 \pm 13.22$, respectively, with no statistically significant difference $(P<0.05)$. Additionally, the age range in all participants was $16-71$ years and $41.7750 \pm 14.3574$ and $44.025 \pm 13.3002$ years in the ginger and diclofenac groups, respectively. The results of this study showed that there was no significant difference regarding the diseases between the two groups $(P>0.05)$. It was not possible to investigate this issue because no side effects such as infection, vomiting, and the like were observed in the patients.

Table 1 demonstrates the results related to pain severity among the groups receiving ginger and diclofenac. Based on the results, no significant difference was found regarding pain severity between the two groups at $0,2,4$, and 6 hours after the recovery $(P>0.05)$.

In addition, the results revealed no significant difference in the duration between recovery and pain improvement between the two groups $(P>0.05)$.

The duration of pain in the two groups is shown in Table 2. Although there was no significant difference between the two groups, most patients experienced pain for 3 hours.

Twenty-five milligrams of pethidine was injected to the patient with a score of higher than 5 or on his/her own request. Accordingly, about 0.12 and 0.1 of the patients in the ginger and diclofenac groups, respectively, received pethidine on their own request or because of the pain score above 5 , with no statistically significant difference $(P>0.05)$.

\section{Discussion}

This study aimed at comparing the effects of ginger capsule and diclofenac tablet on pain relief after inguinal hernia surgery. The severity of pain was not significantly different between the two groups at different intervals after

Table 1. Pain severity in the groups receiving ginger capsule and diclofenac tablet at different intervals

\begin{tabular}{lccc}
\hline \multirow{2}{*}{ Time of study } & \multicolumn{2}{c}{ Group } & \multirow{2}{*}{$\boldsymbol{P}$ value } \\
\cline { 2 - 3 } & Ginger capsule & Diclofenac & \\
\hline Recovery & $5.02 \pm 1.44$ & $4.82 \pm 1.33$ & 0.552 \\
2 h after recovery & $3.17 \pm 1.12$ & $3.05 \pm 1.03$ & 0.608 \\
4 h after recovery & $1.52 \pm 1.10$ & $1.4 \pm 1.03$ & 0.603 \\
6 h after recovery & $0.27 \pm 0.84$ & $0.25 \pm 0.77$ & 0.893 \\
\hline
\end{tabular}

Table 2. Duration of the pain in the groups receiving ginger capsule and diclofenac tablet

\begin{tabular}{lccc}
\hline & \multicolumn{2}{c}{ Group } & \multirow{2}{*}{$\boldsymbol{P}$ value } \\
\cline { 2 - 3 } & Ginger capsule & Diclofenac & \\
\hline Duration of pain $(\mathrm{h})$ & $5.75 \pm 1.12$ & $5.70 \pm 1.15$ & 0.845 \\
\hline
\end{tabular}


the recovery. Further, the pain between the recovery and pain improvement failed to significantly differ between the two groups. Consistent with these results, the positive effects of ginger or its derivatives on various types of pains were confirmed by other studies, including those of Yip et al on knee pain (27), Chen et al on dysmenorrhea (28), Black et al on muscle pain (29), Al-Nahain et al on pain caused by rheumatoid arthritis (30), and Leach et al on osteoarthritis (31).

However, a review article reported that despite the anti-inflammatory effects of ginger, its effect on pain relief remains to be elucidated (24). This can be due to the differences in the methodology of other studies. Additionally, the type of the mental experience of pain, the culture, and the type of pain measurement scale could lead to inconsistency in the findings.

Ginger rhizome has been used for many human diseases such as gastrointestinal in various parts of the world since the ancient times, and its medicinal properties have been often attributed to the presence of phenolic compounds, along with its antioxidant and anti-inflammatory properties $(32,33)$. By containing active ingredients such as gingerol, ginger can reduce inflammation and thus relieve the pain (34). Anthocyanins are glycoside polyhydroxyl derivatives and methoxyl, 2-phenylbenzopyrylium salts, as well as nontoxic and water-soluble pigments that are widely found in nature. The red, blue, purple, amethystine, and black colors in many fruits, vegetables, and flowers are due to the presence of anthocyanins. These compounds are highly potent antioxidants that can suppress the pathways related to the pain by inhibiting cyclooxygenase and lipoxygenase. There are phenolic compounds and anthocyanins such as gingerdion, shoagol, and gingerol in ginger (35). These three compounds are strong prostaglandin inhibitors that prevent the metabolism of arachidonic acid by inhibiting cyclooxygenase and lipoxygenases. In addition, these compounds can impede inflammatory pathways by inhibiting nitric oxide synthase and thus exert their analgesic effects $(36,37)$.

\section{Conclusion}

Overall, no significant difference was observed between ginger and diclofenac in postoperative pain relief, which indicates the acceptable analgesic effect of ginger. Besides, considering the dose of ginger in this study, it can be concluded that higher doses of ginger can even exhibit a greater analgesic effect compared to those of diclofenac if they cause no side effects. In this study, there was no specific side effect in the group receiving ginger and its analgesic effect was similar to that of diclofenac. Therefore, it seems that ginger supplementation can be used to relieve pain with comparatively fewer side effects.

Conflict of Interests

The authors have no conflict of interests.
Ethical considerations

This article was derived from a research project approved by the Research and Technology Deputy of the Shahrekord University of Medical Sciences (approval No. 1964) with the ethics code of IR.SKUMS.REC.1394-75).

\section{Acknowledgments}

This article was obtained from a research project approved by the Research and Technology Deputy of the Shahrekord University of Medical Sciences (approval no.: 1964). Hereby, the authors gratefully thank the women who participated in this study.

\section{References}

1. Duenas M, Salazar A, Ojeda B, Fernandez-Palacin F, Mico JA, Torres LM, et al. A nationwide study of chronic pain prevalence in the general spanish population: identifying clinical subgroups through cluster analysis. Pain Med. 2015;16(4):811-22. doi: 10.1111/pme.12640.

2. Leadley RM, Armstrong N, Lee YC, Allen A, Kleijnen J. Chronic diseases in the European Union: the prevalence and health cost implications of chronic pain. J Pain Palliat Care Pharmacother. 2012;26(4):310-25.doi:10.3109/15360288.2012.736933.

3. Langley PC, Ruiz-Iban MA, Molina JT, De Andres J, Castellon JR. The prevalence, correlates and treatment of pain in Spain. J Med Econ. 2011;14(3):367-80. doi: 10.3111/13696998.2011.583303.

4. Mirjafari SA, Ghaderi H, Kazemeini A, Masoumi G, Shahzamani M, Naimi SS, et al. Is patients' verbal expression of pain intensity reliable? A prospective comparison of three methods of pain assessment by patients, nurses, and physicians. J Negat Results Clin Exp Stud. 2018;1(1):1-5.

5. Duenas M, Ojeda B, Salazar A, Mico JA, Failde I. A review of chronic pain impact on patients, their social environment and the health care system. J Pain Res. 2016;9:457-67. doi: 10.2147/jpr.s105892.

6. Wells N, Pasero C, McCaffery M. Improving the quality of care through pain assessment and management. In: Hughes RG, ed. Patient safety and quality: An evidence-based handbook for nurses. Rockville, MD: Agency for Healthcare Research and Quality; 2008.

7. World Health Organization (WHO). Cancer pain relief: with a guide to opioid availability. WHO; 1996.

8. Landreneau RJ, Mack MJ, Hazelrigg SR, Naunheim K, Dowling RD, Ritter $\mathrm{P}$, et al. Prevalence of chronic pain after pulmonary resection by thoracotomy or video-assisted thoracic surgery. J Thorac Cardiovasc Surg. 1994;107(4):1079-85; discussion 85-6.

9. Garimella V, Cellini C. Postoperative pain control. Clin Colon Rectal Surg. 2013;26(3):191-6. doi: 10.1055/s-0033-1351138.

10. Khodadadi S, Rafieian-Kopaei M. Herbs, health and hazards; a nephrology viewpoint on current concepts and new trends. Ann Res Antioxid. 2016;1(1):1-5.

11. Rafieian-Kopaei M, Baradaran A, Rafieian M. Plants antioxidants: From laboratory to clinic. J Nephropathol. 2013;2(2):152-3. doi: 10.12860/jnp.2013.26.

12. Asgari A. Herbal medicines and kidney; friends or foes? J Nephropharmacol. 2014;3(1):5-6.

13. Jafari T. Antioxidants; helpful or harmful? Ann Res Antioxid. 2016;1(2):1-2.

14. Rafieian-Kopaei M. Medicinal plants for renal injury prevention. J Renal Inj Prev. 2013;2(2):63-5. doi: 10.12861/ jrip.2013.21.

15. Hajian S. Positive effect of antioxidants on immune system. Immunopathol Persa. 2015;1(1):1-2.

16. Baradaran A. Administration of herbal drugs in geriatric individuals; trends on its helps and hazards. Geriatr Persia. 2017;1(1):1-2.

17. Maroon JC, Bost JW, Maroon A. Natural anti-inflammatory 
agents for pain relief. Surg Neurol Int. 2010;1:80. doi: 10.4103/2152-7806.73804.

18. Harrison AM, Heritier F, Childs BG, Bostwick JM, Dziadzko MA. Systematic review of the use of phytochemicals for management of pain in cancer therapy. Biomed Res Int. 2015;2015:506327. doi: 10.1155/2015/506327.

19. Kafeshani M. Ginger, micro-inflammation and kidney disease. J Ren Endocrinol. 2015;1(1):1-2.

20. Nasri H. Herbal drugs and new concepts on its use. J Prev Epidemiol. 2016;1(1):1-2.

21. Rafieian-Kopaei $M$, Nasri H. Ginger and diabetic nephropathy. J Renal Inj Prev. 2013 Mar 1;2(1):9-10.

22. Rahmani AH, Shabrmi FM, Aly SM. Active ingredients of ginger as potential candidates in the prevention and treatment of diseases via modulation of biological activities. Int J Physiol Pathophysiol Pharmacol. 2014;6(2):125-36.

23. Rahnama P, Montazeri A, Huseini HF, Kianbakht $S$, Naseri M. Effect of Zingiber officinale R. rhizomes (ginger) on pain relief in primary dysmenorrhea: a placebo randomized trial. BMC Complement Altern Med. 2012;12:92. doi: 10.1186/14726882-12-92.

24. Terry R, Posadzki P, Watson LK, Ernst E. The use of ginger (Zingiber officinale) for the treatment of pain: a systematic review of clinical trials. Pain Med. 2011;12(12):1808-18. doi: 10.1111/j.1526-4637.2011.01261.x.

25. Noroozinia H, Mahoori A, Hassani E, Akhbari P. Diclofenac suppository versus intramuscular pethidine in post herniorrhaphy pain relief. Tehran University Medical Journal. 2011;69(3):198-203.

26. Kersten P, White PJ, Tennant A. Is the pain visual analogue scale linear and responsive to change? An exploration using Rasch analysis. PLoS One. 2014;9(6):e99485. doi: 10.1371/ journal.pone.0099485.

27. Yip YB, Tam AC. An experimental study on the effectiveness of massage with aromatic ginger and orange essential oil for moderate-to-severe knee pain among the elderly in Hong Kong. Complement Ther Med. 2008;16(3):131-8. doi: 10.1016/j.ctim.2007.12.003.

28. Chen CX, Barrett B, Kwekkeboom KL. Efficacy of oral ginger (zingiber officinale) for dysmenorrhea: a systematic review and meta-analysis. Evid Based Complement Alternat Med. 2016;2016:6295737. doi: 10.1155/2016/6295737.

29. Black CD, Herring MP, Hurley DJ, O'Connor PJ. Ginger (Zingiber officinale) reduces muscle pain caused by eccentric exercise. J Pain. 2010;11(9):894-903. doi: 10.1016/j. jpain.2009.12.013.

30. Al-Nahain A, Jahan R, Rahmatullah M. Zingiber officinale: A potential plant against rheumatoid arthritis. Arthritis. 2014;2014:159089. doi: 10.1155/2014/159089.

31. Leach MJ, Kumar S. The clinical effectiveness of Ginger (Zingiber officinale) in adults with osteoarthritis. Int J Evid Based Healthc. 2008;6(3):311-20. doi: 10.1111/j.17441609.2008.00106.x.

32. Shukla $Y$, Singh M. Cancer preventive properties of ginger: a brief review. Food Chem Toxicol. 2007;45(5):683-90. doi: 10.1016/j.fct.2006.11.002.

33. Rafieian-Kopaei $\mathrm{M}, \mathrm{Nasri} \mathrm{H}$. The Ameliorative Effect of Zingiber officinale in Diabetic Nephropathy. Iran Red Crescent Med J. 2014;16(5):1-2. doi: 10.5812/ircmj. 11324.

34. Wilson PB. Ginger (Zingiber officinale) as an analgesic and ergogenic aid in sport: a systemic review. J Strength Cond Res. 2015;29(10):2980-95. doi: 10.1519/jsc.0000000000001098.

35. Ghasemzadeh A, Jaafar HZ, Rahmat A. Identification and concentration of some flavonoid components in Malaysian young ginger (Zingiber officinale Roscoe) varieties by a high performance liquid chromatography method. Molecules. 2010;15(9):6231-43. doi: 10.3390/molecules15096231.

36. Aktan F, Henness S, Tran VH, Duke CC, Roufogalis BD, Ammit AJ. Gingerol metabolite and a synthetic analogue Capsarol inhibit macrophage NF-kappaB-mediated iNOS gene expression and enzyme activity. Planta Med. 2006;72(8):72734. doi: 10.1055/s-2006-931588.

37. Li F, Nitteranon V, Tang X, Liang J, Zhang G, Parkin KL, et al. In vitro antioxidant and anti-inflammatory activities of 1-dehydro-[6]-gingerdione, 6-shogaol, 6-dehydroshogaol and hexahydrocurcumin. Food Chem. 2012;135(2):332-7. doi: 10.1016/j.foodchem.2012.04.145. 